\title{
Uma adaptação da definição de corpo para o Ensino Fundamental: resolução de equações por meio dos elementos neutro e oposto
}

\author{
Ivana Manfio Cocco (D) Luciane Gobbi Tonet (D)
}

\begin{abstract}
Resumo
Neste trabalho, apresentamos uma proposta didática contemplando o estudo dos conceitos de elemento neutro e oposto em uma turma de Sétimo Ano da Escola Municipal de Ensino Fundamental José Rubin Filho, localizada no município de Pinhal Grande, RS. Para isso, adaptamos a definição de corpo para esse nível de ensino, tendo como suporte a Teoria das Transposições Didáticas, e trabalhamos com a resolução de equações baseada no emprego desses elementos. Abordamos operações distintas das usuais com o objetivo de priorizar um ensino de Álgebra que vai além do uso de regras e técnicas sem significação por parte dos alunos. De acordo com os resultados obtidos, consideramos que a nossa proposta de atividade foi satisfatória e é digna de ser reaplicada. Esse artigo é proveniente da dissertação de mestrado do Profmat - Mestrado Profissional em Matemática em Rede Nacional (COCCO, 2020).
\end{abstract}

Palavras-chave: Corpo; Elemento Neutro e Oposto; Resolução de Equações; Operações Não Usuais.

\section{Abstract}

In this work we presented a didactic proposal that seeks to complete the study of the concepts of neutral and opposite element in a class of Seventh Grade Elementary School, located in the city Pinhal Grande, RS. For this, we adapted of field definition for this level of education, supported by the Theory of Didactic Transpositions, and worked with the solution of equations based on the use of these elements. We approached operations different from the usual ones in order to prioritize teaching Algebra that goes beyond the use of rules and techniques without meaning by the students. According to the results obtained, we believe that our activity proposal was satisfactory and worthy of being reapplied. This article comes from the master's dissertation of PROFMAT - Professional Master's Course in Mathematics.

Keywords: Field; Neutral and Opposite Element; Solving Equation; Unusual Operations.

\section{Introdução}

Na disciplina de Aritmética, presente na matriz curricular do curso de Mestrado Profissional em Matemática em Rede Nacional (Profmat), estudamos a definição de corpo e algumas de suas propriedades. Temos a convicção de que não é possível abordar tal definição no Ensino Básico, 
especialmente da maneira como é apresentada em Cursos Superiores. Entretanto, isso não diminui sua importância nesse nível de ensino, pois por meio dela é possível justificar e compreender propriedades operacionais que, muitas vezes, são estudadas como regras a serem memorizadas.

Desse modo, neste trabalho apresentamos uma sequência didática adaptando a definição de corpo para uma linguagem apropriada ao Ensino Fundamental da Educação Básica. Para isso, julgamos pertinente abordar apenas uma operação, distinta das operações usualmente estudadas neste nível de ensino, no Conjunto dos Números Racionais.

Os documentos norteadores da Educação Básica não mencionam o estudo de operações distintas das usuais, porém Miguel (2005, p.376) assegura que:

\section{[...] o conhecimento matemático não se consolida como um rol de ideias prontas a serem memorizadas; muito além disso, um processo significativo de ensino de Matemática deve conduzir os alunos à exploração de uma grande variedade de ideias e de estabelecimento de relações entre fatos e conceitos [....] (Miguel, 2005, p. 376)}

No entanto, ao analisarmos a Base Nacional Comum Curricular, evidenciamos a importância da Álgebra nos currículos escolares, uma vez que

a unidade temática Álgebra, por sua vez, tem como finalidade o desenvolvimento de um tipo especial de pensamento - pensamento algébrico - que é essencial para utilizar modelos matemáticos na compreensão, representação e análise de relações quantitativas de grandezas e, também, de situações e estruturas matemáticas, fazendo uso de letras e outros símbolos (BRASIL, 2017, p. 270)

Dentro dessa unidade temática, as equações possuem papel de destaque, pois são essenciais na resolução de diversos problemas, sejam eles matemáticos ou da área das Ciências da Natureza. No que diz respeito a sua abordagem na Educação Básica, é comum tratar o ensino de equações de modo mecânico, baseado no uso de regras e macetes de resolução. Tal abordagem pode estar relacionada com a maneira com que os livros didáticos apresentam esse conteúdo, uma vez que, segundo Barbosa e Lins (2013), são esses, muitas vezes, o único recurso pedagógico disponível, desempenhando um papel fundamental no sistema escolar.

No que segue deste artigo, na Seção 2, abordaremos brevemente a Teoria das Transposições Didáticas, a qual fundamenta nossa metodologia de pesquisa. Na sequência, exploramos a definição de corpo, direcionando as propriedades de modo particular para o conjunto dos Números Racionais $\mathbb{Q}$. Destacamos também o estudo de um exemplo contendo uma operação não usual, como forma de explorar as definições de elementos neutro e oposto relativos a tal operação. Na Seção 4, desenvolvemos a sequência didática, contendo cada etapa proposta em aula. Na Seção 5, ilustramos alguns dados coletados na turma em que aplicamos a proposta. Encerramos este artigo com as considerações finais, nas quais destacamos a validade da aplicação da proposta, bem como as possíveis sugestões de alteração para uma próxima aplicação.

Este artigo é proveniente da dissertação de mestrado do Profmat. Para maiores detalhes acerca dos tópicos aqui abordados, indicamos Cocco (2020).

\section{Metodologia da Pesquisa}

A proposta que apresentamos neste artigo baseia-se na teoria das Transposições Didáticas. Esse termo foi introduzido pelo sociólogo Michel Verret (1975), mas aprimorado por Yves Chevallard 
e Marie-Albert Johsua (1982), já no âmbito da Matemática, de onde se expandiu para diversas outras áreas.

Basicamente, designamos transposição didática ao processo de adaptação necessário para transformar um objeto científico em conhecimento escolar, tornando-se uma poderosa ferramenta no que se refere a melhorias das práticas de ensino vigentes. Segundo Neves e Barros (apud Chevallard, 2005 , p. 45)

Um conteúdo do saber que tenha sido designado como saber a ensinar, sofre a partir de então um conjunto de transformações adaptativas que vão torná-lo apto a ocupar um lugar entre os objetos de ensino. Este "trabalho" que transforma um objeto de saber a ensinar em um objeto de ensino é denominado de transposição didática. (Chevallard, 2005, p. 45)

As transposições didáticas são classificadas em transposição didática externa e transposição didática interna, conforme quem as realizam e como as realizam. Mais especificamente, neste artigo, abordamos a transposição didática interna, a qual é realizada pelo professor ao planejar sua aula e resulta no saber ensinado. Conforme Neves e Barros (2011), "vislumbramos a teoria da transposição didática que nos mostra a importância de se pensar no preparo das aulas: como redigi-las, como organizá-las, como contextualizá-las; isso porque, em essência, o trabalho de transposição diz respeito aos saberes."

No que se refere à Matemática, mais especificamente em seus livros didáticos, os conteúdos, por muitas vezes, aparecem desprovidos do seu real significado ou de uma justificativa mais elaborada. Essa simplificação no teor dos conteúdos abordados tem por objetivo tornar o livro didático mais acessível ao estudante. No entanto, em várias situações, tal padrão dificulta ainda mais a aprendizagem desses conteúdos.

Como se observa, o material didático à disposição do Professor do ensino médio difere daquele direcionado ao ensino universitário. Enquanto este último sofreu uma Transposição Didática de fato, o outro pode ser entendido como um processo de simplificação que busca adequar linguagem e recursos matemáticos mínimos para manter o corpo estrutural do Saber a Ensinar. É esse último material didático que o Professor do ensino médio - via de regra - toma como referência para preparar suas aulas. (Alves Filho et al., 2001, p. 10)

A transposição didática surge como uma forma de aliar aos conteúdos apresentados nos livros de Educação Básica toda a base científica comumente expressada em artigos científicos. Neste sentido, o professor faz a transposição didática ao tornar conteúdos estudados a nível da Educação Superior compreensíveis no âmbito da Educação Básica.

Em relação à nossa proposta, selecionamos o conceito de anel, presente na disciplina de Aritmética do curso de Mestrado Profmat e o adaptamos a uma linguagem mais acessível aos alunos do Ensino Fundamental. Basicamente, para esse público-alvo, julgamos pertinente restringirmo-nos a apenas uma nova operação dentro do conjunto dos Números Racionais $\mathbb{Q}$.

Convém ressaltar que, em particular, a transposição didática nos proporcionou a oportunidade de estudarmos as propriedades inerentes a uma operação não usual, isto é, distinta das operações comumente abordadas na Educação Básica, tais como associatividade, comutatividade e existência de elemento neutro e inverso, por exemplo, de forma mais expressiva. Tais propriedades, muitas 
vezes, são abordadas de maneira superficial em livros didáticos frente à dificuldade da demonstração das mesmas perante operações usuais.

\section{O corpo dos Números Racionais}

Recordamos que um conjunto K, munido de duas operações (+) e (·), ditas adição e multiplicação, respectivamente, é chamado anel comutativo com unidade se as seguintes propriedades operacionais forem satisfeitas, para quaisquer $\mathrm{a}, \mathrm{b}, \mathrm{c} \in \mathrm{K}$ :

1. As operações são comutativas, isto é, $a+b=b+a$ e $a \cdot b=b \cdot a$.

2. As operações são associativas, isto é, $a+(b+c)=(a+b)+c$ e a $\cdot(b \cdot c)=(a \cdot b) \cdot c$.

3. Ambas as operações possuem elementos neutros, isto é, existem $0 \in \mathrm{K}$ e $1 \in \mathrm{K}$, tais que, $\mathrm{a}+0=0+\mathrm{a}=\mathrm{a}$ e $\mathrm{a} \cdot 1=1 \cdot \mathrm{a}=\mathrm{a}$.

4. Qualquer elemento de $K$ admite elemento oposto, isto é, para todo a $\in \mathrm{K}$, existe $-\mathrm{a} \in \mathrm{K}$ tal que $\mathrm{a}+(-\mathrm{a})=0$.

5. Valem as leis distributivas da multiplicação em relação à adição, isto é, $a \cdot(b+c)=a \cdot b+a \cdot c e$ $(\mathrm{a}+\mathrm{b}) \cdot \mathrm{c}=\mathrm{a} \cdot \mathrm{c}+\mathrm{b} \cdot \mathrm{c}$.

Se, além dessas propriedades, todo elemento não nulo $b \in \mathrm{K}$ possui um inverso em $\mathrm{K}$, isto é, para todo $\mathrm{b} \neq 0$, existe $\mathrm{b}^{-1} \in \mathrm{K}$ tal que $\mathrm{b} \cdot \mathrm{b}^{-1}=1$, dizemos que $\mathrm{K}$ é um corpo.

A definição acima, da maneira como geralmente nos é apresentada nos cursos de graduação, causa estranheza e é alvo de muitas dificuldades para os estudantes, em parte devido a sua completa abstração e total desconexão com os conhecimentos prévios dos alunos. Devido a isso, no que se refere a sua abordagem na Educação Básica, buscamos trabalhar as propriedades operacionais presentes nesta definição com tranquilidade, para que somente depois desta etapa o uso delas torne-se corriqueiro na disciplina de Matemática, principalmente para a resolução de equações.

Dessa forma, adotamos uma postura antagônica à tradicionalmente proposta em livros didáticos do Ensino Fundamental, nos quais essas propriedades operacionais geralmente são tratadas como regras. Habitualmente, tais livros iniciam a abordagem dessas propriedades por meio de exemplos numéricos e, mesmo havendo um convencimento das suas validades, não são feitas formalizações dos resultados, conforme destaca Colaço (2010, p. 7):

Já os livros didáticos dos tempos atuais têm mostrado ausência, por muitas vezes completas, de explicações de teor matemático para que o aluno esteja a par, e entenda matematicamente, o porquê de certas passagens matemáticas na resolução de problemas que envolvam propriedades das operações. (Colaço, 2010, p. 7)

A abordagem de uma operação distinta da usual, entre outros fatores, colabora com a compreensão da definição de elemento neutro de uma maneira mais efetiva. Ao aluno, nesse caso, propiciamos uma oportunidade para perceber que os números zero e um não são os únicos elementos neutros aditivo e multiplicativo existentes, o que geralmente é alvo de muitas dificuldades em alunos de cursos superiores na área das exatas. A mesma reflexão estende-se à definição de elemento oposto.

Com o propósito de exemplificar nossa proposta, vamos considerar um exercício proposto por Zahn (2013). No Conjunto dos Números Racionais $(\mathbb{Q})$, definimos, para quaisquer $\mathrm{x}, \mathrm{y} \in \mathbb{Q}$, as operações de adição e multiplicação, respectivamente, por

$$
\mathrm{x} * \mathrm{y}=\mathrm{x}+\mathrm{y}-3
$$




$$
x T y=x+y-\frac{1}{3} x y .
$$

Ressaltamos que quando os elementos oposto e inverso referirem-se às operações de adição e multiplicação usuais, respectivamente, suprimiremos a menção a tais operações. Apenas faremos distinção quando as operações não forem as usuais. Além disso, em Cocco (2020, p. 17) apresentamos o procedimento completo para demonstrar que a terna $(\mathbb{Q}, *, T)$ é um corpo. Nesse artigo, abordaremos apenas os cálculos dos elementos neutro e oposto, contemplando nosso tema central.

Sejam a, b, c $\in \mathbb{Q}$ elementos quaisquer. Observamos que,

1) para todo $\mathrm{a} \in \mathbb{Q}, \mathrm{a} * \mathrm{e}=\mathrm{a}$, implica que $\mathrm{a}+\mathrm{e}-3=\mathrm{a}$ e, portanto, adicionando o oposto dos números a e -3 a ambos os membros desta igualdade, concluímos que existe $\mathrm{e}=3 \in \mathbb{Q}$ elemento neutro relativo à operação *.

2) para todo $\mathrm{a} \in \mathbb{Q}, \mathrm{aTe}^{\prime}=\mathrm{a}$ implica que $\mathrm{a}+\mathrm{e}^{\prime}-\frac{1}{3} \mathrm{ae}^{\prime}=\mathrm{a}$. Ou seja, $\mathrm{e}^{\prime}\left(1-\frac{1}{3} \mathrm{a}\right)=0$ e, portanto, $\mathrm{e}^{\prime}=0 \in \mathbb{Q}$ é o elemento neutro relativo à operação $\mathrm{T}$.

3) para todo $\mathrm{a} \in \mathbb{Q}, \mathrm{a} * \mathrm{~s}=\mathrm{e}$, implica que $\mathrm{a}+\mathrm{s}-3=3$. Portanto, adicionando o oposto dos números a e -3 em ambos os membros, concluímos que existe $\mathrm{s}=6-\mathrm{a} \in \mathbb{Q}$ elemento oposto de a.

4) dado $\mathrm{a} \in \mathbb{Q}$ tal que $\mathrm{a} \neq 3$, se $\mathrm{aTr}=0$, então $\mathrm{a}+\mathrm{r}-\frac{1}{3} \mathrm{ar}=0$. Logo, adicionando o oposto do número a em ambos os membros e aplicando a propriedade distributiva, obtemos $\mathrm{r}\left(1-\frac{1}{3} \mathrm{a}\right)=-\mathrm{a}$. Portanto, existe $\mathrm{r}=-\frac{3 \mathrm{a}}{3-\mathrm{a}} \in \mathbb{Q}$ elemento inverso de $\mathrm{a} \neq 3$.

Ressaltamos que apenas faz sentido falar em inverso para elementos não nulos. No nosso contexto, um elemento a $\in \mathbb{Q}$ é não nulo se a $\neq 3$, haja visto que $3 \in \mathbb{Q}$ é o elemento neutro aditivo.

A seguir, apresentamos uma equação cuja resolução está baseada nos conceitos de elemento neutro e oposto de cada uma das operações envolvidas. Nosso objetivo é mostrar que, sem a compreensão efetiva de ambos os conceitos, não podemos resolver as atividades propostas em Cocco (2020, p. 20).

Exemplo Inicial: Resolva a equação $(20 * \mathrm{x}) \mathrm{T} 2=11 \mathrm{em} \mathbb{Q}$.

Primeiramente observamos que, conforme vimos no item 4 , o elemento inverso de $2 \in \mathbb{Q}$ é $\mathrm{r}=$ $-\frac{3.2}{3-2}=-6$. Desse modo, operamos -6 em ambos os membros da equação inicial e, aplicando a propriedade associativa de $\mathrm{T}$, obtemos

$$
\begin{aligned}
{[(20 * \mathrm{x}) \mathrm{T} 2] \mathrm{T}(-6) } & =11 \mathrm{~T}(-6) \\
(20 * \mathrm{x}) \mathrm{T}[2 \mathrm{~T}(-6)] & =11+(-6)-\frac{1}{3} \cdot 11 \cdot(-6) \\
(20 * \mathrm{x}) \mathrm{T} 0 & =27 \\
20 * \mathrm{x} & =27
\end{aligned}
$$

uma vez que 0 é o elemento neutro da operação T.

Novamente, de acordo com o item $3, \mathrm{~s}=6-20=-14$ é o elemento oposto de $20 \in \mathbb{Q}$ em relação à operação *. Dessa forma, operamos com -14 em ambos os membros da igualdade 1 e, por meio 
das propriedades comutativa e associativa de $*$, obtemos

$$
\begin{aligned}
(20 * \mathrm{x}) *(-14) & =27 *(-14) \\
\mathrm{x} *(20 *(-14)) & =27+(-14)-3 \\
\mathrm{x} * 3 & =10 \\
\mathrm{x} & =10
\end{aligned}
$$

pois 3 é o elemento neutro da operação *. Portanto, o Conjunto Solução dessa equação é $S=\{10\}$.

Por meio da resolução dessa equação, evidenciamos a importância dos conceitos de elemento neutro e oposto relativos a cada uma das operações envolvidas. Além disso, salientamos que o emprego dos "atalhos matemáticos", tais como passa para o outro lado com o sinal contrário ou se está multiplicando passa dividindo, torna inviável a resolução de equações desse tipo, uma vez que, em se tratando de operações não usuais, essas regras não possuem sentido. Em função disso, julgamos pertinente a abordagem inicial das equações com operações usuais, sem menção a tais macetes para resolução. Em nossa concepção, os mesmos serão compreendidos no decorrer da prática em sala de aula.

\section{Desenvolvimento da Sequência Didática}

As atividades que propomos neste trabalho foram desenvolvidas no ano de 2019, no decorrer de nove períodos de cinquenta minutos cada, em uma turma de Sétimo Ano, composta por doze alunos, da Escola Municipal de Ensino Fundamental José Rubin Filho, no município de Pinhal Grande - RS.

Optamos por aplicar a proposta em uma turma regular de ensino, no horário de aula. Por essa razão, estávamos sujeitos a diversas situações imprevistas, como, por exemplo, dificuldades básicas de aprendizagem em Matemática, ausências e indisciplina dos alunos. Dividimos a sequência didática em três partes, que apresentaremos a seguir.

\subsection{Primeira Parte - Introdução às operações não usuais}

A primeira atividade proposta tem como objetivo proporcionar o contato inicial com operações distintas das usuais, ou seja, permitir que os alunos desenvolvam cálculos envolvendo operações diferentes das que estão habituados a trabalhar. No que segue, juntamente com o enunciado da questão, apresentamos uma solução para a mesma.

Questão 1. (Obmep - $2^{\mathrm{a}}$ fase - 2018 - Nível 3 -Adaptada) Sérgio inventou as operações matemáticas $\otimes$ e @ entre os números racionais, como abaixo:

$$
\begin{aligned}
& a \Delta b=a^{2}+b^{2} \\
& a @ b=(a+b)^{2} .
\end{aligned}
$$

Por exemplo, $1 \otimes 4=1^{2}+4^{2}=17$ e $1 @(-6)=(1+(-6))^{2}=25$. Utilizando as operações criadas por Sérgio, responda as questões a seguir:

a) Qual é o valor de:

- $(-1) \otimes 1$ ? 
Solução: $\quad(-1) \otimes 1=(-1)^{2}+1^{2}=1+1=2$.

- $1 \otimes(-5)$ ?

Solução: $1 \otimes(-5)=1^{2}+(-5)^{2}=1+25=26$.

- $2 \otimes 3$ ?

Solução: $2 \otimes 3=2^{2}+3^{2}=4+9=13$.

- $2 @ 0$ ?

Solução: $2 @ 0=(2+0)^{2}=2^{2}=4$.

-3@2?

Solução: $3 @ 2=(3+2)^{2}=5^{2}=25$.

-2@3?

Solução: $2 @ 3=(2+3)^{2}=5^{2}=25$.

b) Qual é o valor de $(2 @ 3)-(2 \otimes 3)$ ?

Solução: $(2 @ 3)-(2 \otimes 3)=(2+3)^{2}-\left(2^{2}+3^{2}\right)=5^{2}-2^{2}-3^{2}=12$.

Em seguida, na segunda atividade, buscamos ilustrar a validade da propriedade comutativa por meio de alguns exemplos numéricos. Ressaltamos que, matematicamente, não podemos concluir que uma operação é comutativa a partir de um número finito de exemplos. Entretanto, essa prática está de acordo com a abordagem geralmente feita nos livros didáticos para esse nível de ensino.

Questão 2. Sejam $a, b \in \mathbb{Q}-\{0\}$. Definimos as operações:

$$
\begin{aligned}
\mathrm{a} \Delta \mathrm{b} & =\mathrm{ab} \\
\mathrm{a} \boldsymbol{b} & =\mathrm{b}^{\mathrm{a}} .
\end{aligned}
$$

- Calcule:

\section{a) $1 \mathbf{\Delta} 3 ?$}

Solução: $1 \mathbf{\Delta} 3=1 \cdot 3=3$.

b) $3 \mathbf{\Delta} 1 ?$

Solução: $3 \mathbf{\Lambda} 1=3 \cdot 1=3$.

c) $(-2) \mathbf{\Delta}(-3) ?$

Solução: $(-2) \boldsymbol{\Lambda}(-3)=(-2) \cdot(-3)=6$.

d) $(-3) \boldsymbol{\Lambda}(-2)$ ?

Solução: $(-3) \boldsymbol{\Delta}(-2)=(-3) \cdot(-2)=6$.

e) $3 \mathbf{\wedge} 2 ?$

Solução: $3 \mathbf{\Lambda} 2=3 \cdot 2=6$.

f) $2 \mathbf{\Delta} 3 ?$

Solução: $2 \mathbf{\wedge} 3=2 \cdot 3=6$. 


\section{g) $3 \triangleleft 5$ ?}

Solução: $3 \triangleleft 5=5^{3}=125$.

h) $2 \checkmark(-1)$ ?

Solução: 2 \} ( - 1 ) = ( - 1 ) ^ { 2 } = 1 \text { . }

i) $6 \downarrow 1$ ?

Solução: $6 \downarrow 1=1^{6}=1$.

j) $1 \triangleleft 6 ?$

Solução: $1 \triangleleft 6=6^{1}=6$.

- Nos itens (i) e (j), realizamos a operação $\bullet$ com os mesmos números; entretanto os resultados obtidos são distintos. O que isso nos permite concluir?

Solução: A operação não é comutativa, pois quando mudamos a ordem das parcelas obtemos resultados diferentes.

- E quanto à operação $\boldsymbol{\Delta}$ : é comutativa? Justifique.

Solução: Nos pares de itens (a) e (b), (c) e (d) e (e) e (f), realizamos a operação $\boldsymbol{\Delta}$ com os mesmos números e obtemos os mesmos resultados. Com isso, temos que, ao mudar a ordem das parcelas, o resultado não se altera. Portanto, a operação ^ é comutativa.

Por fim, abordamos na terceira atividade a generalização da validade de algumas propriedades com relação à determinada operação.

Questão 3. Em $\mathbb{Q}$, definimos a operação:

$$
\mathrm{x} * \mathrm{y}=\mathrm{x}+\mathrm{y}-3
$$

a) Calcule:

- $2 * 3$

Solução: $2 * 3=2+3-3=2$.

- $5 * 3$

Solução: $5 * 3=5+3-3=5$.

- $10 * 3$

Solução: $10 * 3=10+3-3=10$.

- $\frac{8}{5} * 3$

Solução: $\frac{8}{5} * 3=\frac{8}{5}+3-3=\frac{8}{5}$.

b) O que ocorre quando uma das parcelas é 3 ?

Solução: Quando operamos um número com 3, obtemos como resultado o próprio número.

c) Podemos dizer que 3 é o elemento neutro da operação $*$ ?

Solução: Sim, pois para qualquer $\mathrm{a} \in \mathbb{Q}$, temos que $\mathrm{a} * 3=\mathrm{a}+3-3=\mathrm{a}+0=\mathrm{a}$. 
d) Calcule:

- $2 * 4$

Solução: $2 * 4=2+4-3=3$.

- $8 *(-2)$

Solução: $8 *(-2)=8+(-2)-3=3$.

- $3 * 3$

Solução: $3 * 3=3+3-3=3$.

- $5 * 1$

Solução: $5 * 1=5+1-3=3$.

e) Em cada uma das operações realizadas no item (d), obtivemos como resultado o elemento neutro relativo à operação *. O que isso significa?

Solução: Em cada item, um dos números é o oposto do outro.

f) Dado um número a $\in \mathbb{Q}$, qual é o elemento oposto de a?

Solução: Queremos encontrar $\mathrm{a}^{\prime} \in \mathbb{Q}$, tal que a $* \mathrm{a}^{\prime}=3$, ou seja,

$$
\begin{aligned}
a+a^{\prime}-3 & =3 \\
a+a^{\prime}-3+3 & =3+3 \\
a+a^{\prime} & =6 \\
a+a^{\prime}+(-a) & =6+(-a) \\
a^{\prime} & =6-a .
\end{aligned}
$$

Logo, o elemento oposto de a é $\mathrm{a}^{\prime}=6-\mathrm{a} \in \mathbb{Q}$.

g) Calcule:

- $4 * 5$

Solução: $4 * 5=4+5-3=6$.

- $5 * 4$

Solução: $5 * 4=5+4-3=6$.

- $(-2) * 6$

Solução: $(-2) * 6=(-2)+6-3=1$.

- $6 *(-2)$

Solução: $6 *(-2)=6+(-2)-3=1$.

h) Analisando o item anterior, vimos que podemos comutar as parcelas da operação *, e o resultado permanece igual. Será que isso ocorre para quaisquer números?

Solução: Sim. Mostraremos, para o caso geral, que a operação $*$ é comutativa, isto é $\mathrm{a} * \mathrm{~b}=\mathrm{b} * \mathrm{a}$. 
Para isso, consideremos $\mathrm{a}, \mathrm{b} \in \mathbb{Q}$. Assim,

$$
\begin{aligned}
\mathrm{a} * \mathrm{~b} & =\mathrm{a}+\mathrm{b}-3 \\
& =b+a-3 \\
& =b * a .
\end{aligned}
$$

i) Calcule:

- $(1 * 6) * 5$

Solução: $(1 * 6) * 5=(1+6-3) * 5=4 * 5=4+5-3=6$.

- $1 *(6 * 5)$

Solução: $1 *(6 * 5)=1 *(6+5-3)=1 * 8=1+8-3=6$.

- $(9 * 4) * 2$

Solução: $(9 * 4) * 2=(9+4-3) * 2=10 * 2=10+2-3=9$.

- $9 *(4 * 2)$

Solução: $9 *(4 * 2)=9 *(4+2-3)=9 * 3=9$, pois 3 é o elemento neutro da operação $*$.

j) Pelo item anterior, vimos que podemos associar de maneiras distintas as parcelas da operação *, e mesmo assim obtivemos o mesmo resultado. Isso ocorre para quaisquer números racionais?

Solução: Sim. Mostraremos, para o caso geral, que a operação * é associativa, isto é, $(\mathrm{a} * \mathrm{~b}) * \mathrm{c}=$ $\mathrm{a} *(\mathrm{~b} * \mathrm{c})$. Para isso, consideremos $\mathrm{a}, \mathrm{b}, \mathrm{c} \in \mathbb{Q}$. Temos que

$$
\begin{aligned}
(\mathrm{a} * \mathrm{~b}) * \mathrm{c} & =(\mathrm{a}+\mathrm{b}-3) * \mathrm{c} \\
& =(\mathrm{a}+\mathrm{b}-3)+\mathrm{c}-3 \\
& =\mathrm{a}+\mathrm{b}+\mathrm{c}-6 .
\end{aligned}
$$

Por outro lado,

$$
\begin{aligned}
a *(b * c) & =a *(b+c-3) \\
& =a+(b+c-3)-3 \\
& =a+b+c-6 .
\end{aligned}
$$

Portanto, $(\mathrm{a} * \mathrm{~b}) * \mathrm{c}=\mathrm{a} *(\mathrm{~b} * \mathrm{c})$.

Destacamos que os itens (c), (f), (h) e (j) devem ser resolvidos exclusivamente pelo professor na lousa, pois os mesmos consistem em generalizações das propriedades. Na nossa proposta, nesse nível de ensino, não almejamos tornar essas generalizações um exercício destinado ao aluno.

\subsection{Segunda Parte - Resolução de equações}

Dedicamos esta etapa da proposta para o estudo e resolução de um exemplo. Todas as equações foram exploradas com o auxílio do professor, com o objetivo de familiarizar o aluno e preparálo para a próxima etapa. Conforme destacamos nas soluções apresentadas, desenvolvemos uma espécie de roteiro de resolução, elaborado a partir da maneira como os alunos estavam habituados a resolver equações do primeiro grau com uma incógnita. 
Exemplo: Considerando a operação $\mathrm{x} * \mathrm{y}=\mathrm{x}+\mathrm{y}-3$, determine o conjunto solução das equações:

a) $\mathrm{x} * 12=14$.

Solução: Determinamos, inicialmente, o elemento neutro e $\in \mathbb{Q}$ da operação *. Por definição $\mathrm{a} * \mathrm{e}=\mathrm{a}$, ou seja, $\mathrm{a}+\mathrm{e}-3=\mathrm{a}$, para todo $\mathrm{a} \in \mathbb{Q}$. Adicionando o oposto dos números -3 e a em ambos os membros, obtemos $\mathrm{e}=3$, o elemento neutro relativo a operação $*$.

$\mathrm{Na}$ sequência, determinamos o elemento oposto de a $\in \mathbb{Q}$ com relação à operação *. Com efeito, seja $a^{\prime} \in \mathbb{Q}$ o elemento oposto de a. Por definição, $a * a^{\prime}=e$, ou seja, $a+a^{\prime}-3=3$. Adicionando o oposto dos números -3 e a a ambos os membros, concluímos que $\mathrm{a}^{\prime}=6-\mathrm{a}$.

A partir de agora, prosseguimos com a resolução da equação. Para isso, observamos que o elemento oposto de 12 em relação a operação $*$ é $6-12=-6$. Logo,

$$
(\mathrm{x} * 12) *(-6)=14 *(-6) .
$$

Pela associatividade da operação *,

$$
\mathrm{x} *(12 *(-6))=\mathrm{x} * 3=14+(-6)-3=5 .
$$

Como 3 é o elemento neutro da operação $*$, concluímos que $\mathrm{x}=5$. Portanto, o conjunto solução dessa equação é $\mathrm{S}=\{5\}$.

Por fim, achamos oportuno verificar se o resultado obtido está correto, tendo em vista a possível dificuldade que os alunos poderiam apresentar relacionadas ao contexto novo das operações introduzidas. Para isso, devemos substituir na equação inicial a incógnita pelo valor encontrado e obter como resultado o número 14 . Ou seja,

$$
5 * 12=5+12-3=14 .
$$

b) $2 \mathrm{x} * 12=7 * \mathrm{x}$.

Solução: Como vimos anteriormente, o elemento oposto do número 12 em relação à operação * é $6-12=-6$. Desse modo, quando operamos com o número -6 em ambos os membros da equação em questão, obtemos

$$
(2 \mathrm{x} * 12) *(-6)=(7 * \mathrm{x}) *(-6)
$$

Devido às propriedades comutativa e associativa da operação *, segue que

$$
2 \mathrm{x} *(12 *(-6))=(7 *(-6)) * \mathrm{x}
$$

ou seja, $2 \mathrm{x} * 3=(-2) * \mathrm{x}$. Logo, $2 \mathrm{x}=(-2) * \mathrm{x}$

Agora, o elemento oposto do número x relativo a operação * é 6-x. Então, operando com o número 6 - x em ambos os lados da equação anterior e pelas propriedades da operação *, temos

$$
2 \mathrm{x} *(6-\mathrm{x})=(-2) *(\mathrm{x} *(6-\mathrm{x})) .
$$

Ou seja, $\mathrm{x}+3=-2$. Por fim, adicionando o oposto do número 3 nos dois membros dessa equação, concluímos que $\mathrm{x}=-5$. Portanto, o conjunto solução dessa equação é $\mathrm{S}=\{-5\}$. 
Novamente, vamos verificar se a solução encontrada está correta. Para isso, devemos substituir a incógnita pelo valor encontrado em cada membro da equação inicial e obter o mesmo resultado em ambas as substituições. Ou seja,

$$
2 \cdot(-5) * 12=(-10) * 12=-10+12-3=-1 \text {. }
$$

E, por outro lado,

$$
7 *(-5)=7+(-5)-3=-1 \text {. }
$$

\subsection{Terceira Parte - Exercícios resolvidos pelos alunos}

Nesta etapa, os alunos resolveram quatro equações envolvendo uma operação distinta das usuais, baseadas nos exemplos que trabalhamos na etapa anterior. Para isso, eles estavam organizados em trios ou duplas e podiam consultar o material que foi desenvolvido nas aulas anteriores.

Exercício: Considerando operação $\mathrm{x} * \mathrm{y}=\mathrm{x}+\mathrm{y}-3$, resolva as equações:

a) $5 * \mathrm{~m}=0$

Solução: Conforme exemplos explorados na Seção 3 , temos que $6-5=1$ é o elemento oposto de 5 , relativo à operação *. Assim, pela associatividade de *,

$$
\begin{aligned}
1 *(5 * \mathrm{~m}) & =1 * 0 \\
(1 * 5) * \mathrm{~m} & =1+0-3 \\
3 * \mathrm{~m} & =-2 \\
\mathrm{~m} & =-2,
\end{aligned}
$$

pois 3 é o elemento neutro da operação *. Portanto, o conjunto solução é $\mathrm{S}=\{-2\}$.

b) $\mathrm{x} * 23=25$.

Solução: Novamente, conforme exemplos explorados na Seção 3, o elemento oposto de 23 em relação à operação $*$ é $6-23=-17 \in \mathbb{Q}$. Desse modo, pela propriedade associativa de $*$,

$$
\begin{aligned}
(\mathrm{x} * 23) *(-17) & =25 *(-17) \\
\mathrm{x} *(23 *(-17)) & =25+(-17)-3 \\
\mathrm{x} * 3 & =5 \\
\mathrm{x} & =5,
\end{aligned}
$$

uma vez que 3 é o elemento neutro da operação *. Portanto, o conjunto solução é $S=\{5\}$.

c) $(\mathrm{x}-7) * 5=15$

Solução: De acordo com a letra a), o elemento oposto de 5 é $6-5=1 \in \mathbb{Q}$. Assim, pela propriedade associativa de $*$,

$$
\begin{aligned}
{[(\mathrm{x}-7) * 5] * 1 } & =15 * 1 \\
(\mathrm{x}-7) *(5 * 1) & =15+1-3 \\
(\mathrm{x}-7) * 3 & =13 \\
\mathrm{x}-7 & =13
\end{aligned}
$$


pois 3 é o elemento neutro da operação *. Então, adicionando o oposto do número -7 em ambos os membros, concluímos que $\mathrm{x}=20$. Portanto, $\mathrm{S}=\{20\}$.

d) $(\mathrm{m}+4) *(\mathrm{~m}+2)=3$

Solução: Conforme a Seção 3 , o elemento oposto do número $\mathrm{m}+2$ relativo à operação $*$ é $6-(\mathrm{m}+2)=$ $4-\mathrm{m} \in \mathbb{Q}$. Desse modo, pela associatividade de $*$,

$$
\begin{aligned}
{[(\mathrm{m}+4) *(\mathrm{~m}+2)] *(4-\mathrm{m}) } & =3 *(4-\mathrm{m}) \\
(\mathrm{m}+4) *[(\mathrm{~m}+2) *(4-\mathrm{m})] & =4-\mathrm{m} \\
(\mathrm{m}+4) * 3 & =4-\mathrm{m} \\
\mathrm{m}+4 & =4-\mathrm{m},
\end{aligned}
$$

pois 3 é o elemento neutro da operação *. Assim, adicionando os opostos dos números $-\mathrm{m}$ e 4 em ambos os membros, obtemos $2 \mathrm{~m}=0$ e, então, concluímos que $\mathrm{m}=0$. Portanto, o conjunto solução é $\mathrm{S}=\{0\}$.

Na próxima seção, ilustraremos algumas das resoluções destas equações apresentadas pelos alunos.

\section{Sobre Alguns Resultados Obtidos}

Inicialmente, consideremos a equação $5 * \mathrm{~m}=0$. Na Figura 1, apresentamos a resolução dada por um aluno. Primeiramente, o aluno calculou o elemento oposto do número 5 em relação à operação *. Em seguida, utilizou as propriedades associativa e comutativa da operação *. Por fim, na coluna da direita, realizou o processo de verificação, para ter certeza de que o resultado encontrado estava correto.
a) $5 * m=0$
Recemento opOS to $D E 5=6-5=1$
$(5 * M) * 1=0 * 1$
Processo
De
VERIF́CAGAO =
$M *(5 * 1)=0+1-3$
$M *(5+1-3)=-2$
$M * 3=-2$

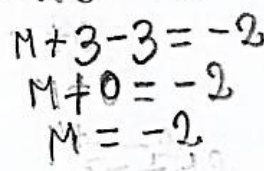

$$
\begin{aligned}
& 5 * M=0 \\
& 5 *(-2)=0 \\
& 5+(-2-3)=0 \\
& 5-5=0 \\
& 0=0 \quad(y)
\end{aligned}
$$

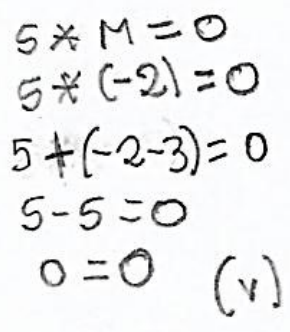

Figura 1: Resposta dada para a equação $5 * \mathrm{~m}=0$.

Com relação à equação $\mathrm{x} * 23=25$. Na Figura 2, apresentamos a resposta obtida por um aluno. Similarmente à resolução anterior, o aluno calculou o elemento oposto do número 23 em relação à operação *. Em seguida, aplicou a propriedade associativa da operação *. Por fim, escreveu o conjunto solução da equação. 
PROFESSOR DE

MATEMÁTICA

Cocco e Tonet

b) $x * 23,=25$.

$$
\begin{aligned}
& \begin{array}{l}
6-23=17-17=25 *-17 \\
x * 235 *-17=2
\end{array} \\
& x *(23 *-17)=25+(-17)-3 \\
& x *(23+(-17-3)=8-3 \\
& x *(23+(-20)=5 \\
& x+2 \geq 5 \\
& x+3=3=5 \\
& x+5=5 \\
& x=5 \\
& S=\{5\}
\end{aligned}
$$

Figura 2: Resposta dada para a equação $\mathrm{x} * 23=25$

Na Figura 3, ilustramos a resolução apresentada por um aluno para a equação $(\mathrm{x}-7) * 5=15$. Inicialmente, o aluno calculou o elemento oposto do número 5 em relação à operação * e aplicou a propriedade associativa dessa operação. Em seguida, realizou o processo de verificação. Alguns alunos tiveram dificuldade em interpretar o elemento $\mathrm{x}-7$ como um único número.

玉tar

$$
\begin{aligned}
& (x-7) * 5=15 d 6=6-5=1 \\
& (x-7) * 5 * 2=25 * 2 \\
& (x-4) *(5 * 6)=25 * 2 \\
& (x-7) *(5+2-5)=25+1-3 \\
& (x-7) * 3=23 \\
& x-7=13 \\
& x=23+7 \\
& x=20
\end{aligned}
$$

Prociszo de Verificasa

$$
\begin{gathered}
(x-7) * 5=15 \\
(20-7) * 5=16 \\
13+5-3=25 \\
+8-3=45 \\
15=25
\end{gathered}
$$

Figura 3: Resposta dada para a equação $(\mathrm{x}-7) * 5=15$.

Na Figura 4, destacamos a resolução desenvolvida por um aluno para a equação $(m+4) *(m+2)=3$. Similarmente às resoluções anteriores, o aluno calculou o elemento oposto de $\mathrm{m}+2$ em relação à operação * e aplicou a propriedade associativa dessa operação. Para finalizar, realizou o processo de verificação. Nessa questão, os alunos também apresentaram dificuldade em perceber que os elementos $\mathrm{m}+4$ e $\mathrm{m}+2$ representavam, cada um deles, um único número. Em consequência disso, não conseguiam determinar de qual dos dois números deveria ser calculado o elemento oposto, uma vez que, em qualquer escolha, tal elemento teria a variável junto.

61

SBM 
d) $(m+4) *(m+2)=3$

$$
\begin{aligned}
& 6-|m+2|=6-m-2=4-m \\
& (m+4) *(m+2)+(4 m)=(4-m) * 3 \\
& (m+4) *[m+2+4-m-3]=4-m+3-3 \\
& \begin{array}{cc}
m+4+3-3-4-m & (m+4) *(m+2)=3 \\
2 m+m=-4-3+3+4 & (0+4) * t+2+2)=3 \\
2 m=-7+\frac{1}{2} & 4+2=3 \\
m=0 & 6-3=3
\end{array}
\end{aligned}
$$

Figura 4: Resposta dada para a equação $(m+4) *(m+2)=3$.

Ao analisarmos todas as resoluções apresentadas pelos alunos, evidenciamos que a maioria deles, mesmo sabendo que o número 3 era o elemento neutro da operação *, refizeram os cálculos envolvendo tal elemento. Apresentamos, na Tabela 1 a seguir, o número de alunos que realizou essa passagem diretamente, utilizando apenas o conceito de neutralidade do número 3 , bem como o número de alunos que refez os cálculos, demonstrando uma possível defasagem na compreensão deste conceito.

\begin{tabular}{|c|c|c|}
\hline Equação & Uso adequado do conceito & Refizeram os cálculos \\
\hline \hline $5 * \mathrm{~m}=0$ & 03 & 09 \\
\hline $\mathrm{x} * 23=25$ & 01 & 11 \\
\hline$(\mathrm{x}-7) * 5=15$ & 07 & 05 \\
\hline$(\mathrm{m}+4) *(\mathrm{~m}+2)=3$ & 03 & 09 \\
\hline
\end{tabular}

Tabela 1: Relação de alunos que utilizaram diretamente o conceito de elemento neutro.

De modo geral, os alunos não apresentaram maiores dificuldades na resolução das atividades, conforme podemos observar na Tabela 2 a seguir.

\begin{tabular}{|c|c|c|c|}
\hline Equação & Acertos & Equívocos & Percentual de acertos \\
\hline \hline $5 * \mathrm{~m}=0$ & 12 & 00 & 100 \\
\hline $\mathrm{x} * 23=25$ & 09 & 03 & 75 \\
\hline$(\mathrm{x}-7) * 5=15$ & 11 & 01 & 91,67 \\
\hline$(\mathrm{m}+4) *(\mathrm{~m}+2)=3$ & 11 & 01 & 91,67 \\
\hline
\end{tabular}

Tabela 2: Relação de acertos e equívocos de cada equação.

Apresentamos, na Figura 5, algumas opiniões dos alunos sobre as atividades que foram desenvolvidas. 

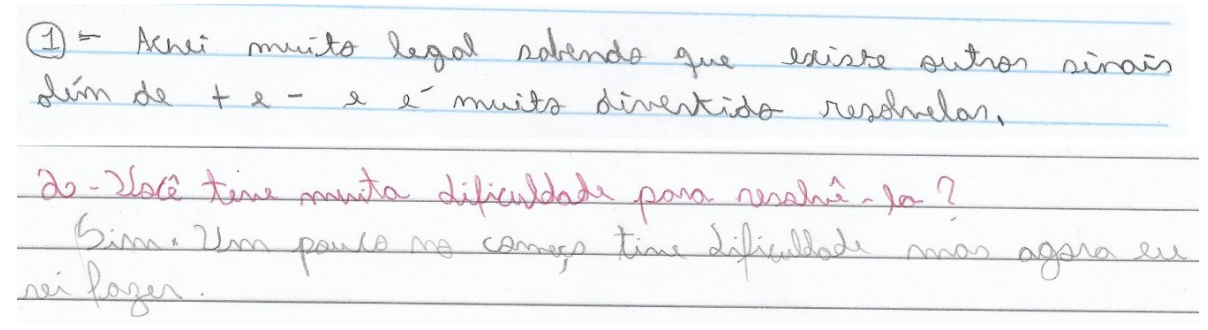

Figura 5: Opiniões sobre as atividades.

Desse modo, através dos relatos dados pelos alunos, constatamos que os mesmos sentiram-se motivados a trabalhar com operações não usuais, uma vez que, apesar de não considerarem as atividades fáceis, afirmaram que foi divertido trabalhar com elas e, além disso, demonstraram que conseguiram compreender o que foi proposto a eles.

\section{Considerações Finais}

Antes de aplicarmos as atividades com os alunos, não trabalhamos a resolução de equações do $1^{0}$ grau com uma incógnita por meio de "atalhos matemáticos". Ao invés disso, enfatizamos o estudo e o emprego dos conceitos dos elementos neutro e oposto das operações de adição e multiplicação usuais. Em virtude disso, os alunos não estavam acostumados a utilizar os tradicionais macetes para isolar a incógnita, tais como "passa para o outro lado com o sinal contrário" ou ainda "se está multiplicando passa para o outro lado dividindo". Acreditamos que a maneira como abordamos esse conteúdo inicialmente foi essencial para que a turma conseguisse resolver as equações que envolviam operações distintas.

Tendo em vista os resultados que obtivemos com as atividades, consideramos que nossa proposta de aplicação é digna de ser repetida em outras oportunidades. Porém, julgamos necessário ampliar o número de exercícios introdutórios para que os alunos possam compreender mais amplamente as definições de elemento neutro e oposto, sem atrelar apenas aos números zero e um.

Além disso, consideramos importante a ampliação do número de exemplos de resoluções de equações propostas para que o emprego dos elementos neutros seja mais efetivo, evitando, assim, que os alunos refaçam os cálculos que envolvem tais elementos.

\section{Referências}

[1] Alves, J. P., Pinheiro, T. F. e Pietrocola, M. A Eletrostática Como Exemplo de Transposição Didática. Ensino de Física: conteúdo metodologia e epistemologia em uma concepção integradora, 2001. Disponível em: <https://edisciplinas.usp.br/pluginfile.php/4370704/mod_resource/ content/5/Textos_para_Resenha/ELETROST\%C3\%81TICA-TDcorrigidopelaTe.pdf/>. Acesso em: 27 de setembro de 2021.

[2] Barbosa, E. J. T. e Lins, A. F. "Equações Polinomiais do Primeiro Grau em Livros Didáticos: Organizações Matemática e Didática". Revista Educação Matemática Pesquisa: Revista do Programa de Estudos Pós-Graduados em Educação Matemática. São Paulo, v. 15, n. 2, p. 337 - 357 , mai./ago. 2013. Disponível em: <https://revistas.pucsp.br/emp/article/view/15062/pdf>. Acesso em: 27 de setembro de 2021 . 
[3] Brasil. Base Nacional Comum Curricular. Brasília: MEC, 2017. Disponível em: $\quad<$ http://portal.mec.gov.br/index.php?option=com_docman\&view=download\&alias= 79601-anexo-texto-bncc-reexportado-pdf-2\&category_slug=dezembro-2017-pdf\&Itemid=30192>. Acesso em: 27 de setembro de 2021.

[4] COCCO, I. M. Explorando os conceitos de elemento neutro e oposto por meio da resolução de equações no Conjunto dos Números Racionais. 2020. 54p. Dissertação (Mestrado Profissional em Matemática em Rede Nacional) - Universidade Federal de Santa Maria, Santa Maria, 2020.

[5] Colaço, W. S. Movimento da Matemática Moderna aos Tempos Modernos: Uma Análise de Livros Didáticos Sobre Explicitação e Exploração das Propriedades de Operações. 2010. 75p. Dissertação (Mestrado Profissional em Ensino de Ciências e Matemática) - Universidade Estadual da Paraíba, Campina Grande, 2010. Disponível em: <http://tede.bc.uepb.edu.br/jspui/ bitstream/tede/1977/1/PDF\%20-\%20Walber\%20Santiago\%20Colaco.pdf $>$. Acesso em: 27 de setembro de 2021.

[6] Miguel, J. C. O Ensino de Matemática na Perspectiva da Formação de Conceitos: Implicações Teórico-Metodológicas. In: Sheila Zambello de Pinho; José Roberto Corrêa Saglietti. (Org.). Núcleos de Ensino - PROGRAD - UNESP. 1 ${ }^{\text {a }}$ ed. São Paulo: UNESP, 2005, v. 1, p. 375-394. Disponível em: <http://www.gradadm.ifsc.usp.br/dados/20121/SLC0630-1/ Ensino-Matematica-Enfoque-Conceitos.pdf>. Acesso: 27 de setembro de 2021.

[7] Neves, K. C. R. e Barros, R. M. O. Diferentes Olhares Acerca da Transposição Didática. Investigações em Ensino de Ciências - V16(1), pp. 103-115, 2011. Disponível em: <file:///C:/Users/bruno/OneDrive/\%C3\%81rea\%20de\%20Trabalho/luciane/UFSM/profmat/ivana/ transposi $\%$ C3\%A7\%C3\%A30\%20did\%C3\%A1tica/249-487-1-SM.pdf>. Acesso: 27 de setembro de 2021.

[8] OBMEP. Banco de questões. Disponível em: <https://drive.google.com/file/d/ 1 bs3rEHm3sE0ELp1N-oFK7QsR2nDLiUEU/view>. Acesso em 27 de setembro de 2021.

[9] Zahn, M. Introdução à Álgebra. Rio de Janeiro: Ciência Moderna, 2013.

Ivana Manfio Cocco Escola Estadual de Ensino Fundamental Joaquim José da Silva Xavier <ivanamanfiococco@hotmailcom>

Luciane Gobbi Tonet Universidade Federal de Santa Maria <lucianegobbi@yahoo.com.br>

Recebido: 30/09/2021

Publicado: 16/02/2022 